\title{
BRAIN TUMORS
}

\section{ADVERSE EFFECTS OF RADIATION AND CHEMOTHERAPY}

The long-term consequences of radiation and chemotherapy on intellectual and endocrine function in children with brain tumors is reviewed from the Departments of Neurology and Pediatrics, State University of New York, Buffalo, NY. The young age at time of radiation, high doses of radiation, site of the tumor, and use of methotrexate are risk factors for the development of both endocrinopathies and intellectual dysfunction. Delay in the use of radiation and the substitution of post-operative chemotherapy in patients younger than 3 years of age may delay development of intellectual dysfunction in surviving children. The omission of methotrexate from treatment programs may avoid the complications of leukoencephalopathy and dementia, although the influence of other chemotherapeutic agents on intellectual functioning is as yet unknown. All Pediatric Oncology Group protocols currently include base-line neuropsychologic evaluations as well as yearly follow-up exams throughout the course of study. Improved quality of life is a primary goal of treatment regimens. (Duffner PK, Cohen ME. Longterm consequences of CNS treatment for childhood cancer, part II: clinical consequences. Pediatr Neurol July/Aug 1991; $\underline{7}: 237-242$ ).

COMMENT. The increase in survival rate of children with brain tumors over the past 20 years has been accompanied by a decrease in intellectual functioning and other serious complications. The recognition of risk factors for long-term adverse consequences of radiation and chemotherapy should lead to modifications of treatment regimens and improvement in academic potential.

In a study at the Children's Hospital and Harvard Medical School, Boston, MA, comparing the effectiveness of thallium-201 SPECT imaging with MRI in tumor assessment, SPECT allowed a more precise assessment of the functional state of the tumor but was less reliable than the MRI in detecting possible radiation injury in some patients (O'Tuama LA et al. SPECT imaging of treated childhood brain tumors. Pediatr Neurol 1991; $\underline{7}: 249-257)$.

Green DM et al, at the Roswell Park Cancer Institute, Buffalo, NY, report that the frequency of congenital anomalies was not increased in children of men or women who had received chemotherapy for cancer during childhood and adolescence, with the possible exception of those treated with dactinomycin, at increased risk for cardiac defects (N Engl J Med July 18,$1991 ; \underline{325}: 141-6)$.

\section{PROGNOSIS OF BRAIN TUMOURS WITH EPILEPSY}

The prognosis of 560 patients with a clinical and CT diagnosis of intrinsic supratentorial brain tumour was examined retrospectively at the Department of Neurosciences, Walton Hospital, Liverpool, England. Epilepsy was the first symptom in 164 patients. Patients presenting with epilepsy had 